\title{
Mitophagy Failure in APP and Tau Overexpression Model of Alzheimer's Disease
}

\author{
Patricia Martín-Maestro ${ }^{\mathrm{a}, \mathrm{b}, 1}$, Ricardo Gargini ${ }^{\mathrm{a}, \mathrm{c}}$, Esther García ${ }^{\mathrm{a}}$, Diana Simón ${ }^{\mathrm{d}}$, Jesús Avila a,b,* \\ and Vega García-Escudero, a,b,2,* \\ ${ }^{a}$ Centro de Biología Molecular "Severo Ochoa” (UAM-CSIC), Cantoblanco, Madrid, Spain \\ ${ }^{\mathrm{b}}$ Centro de Investigación Biomédica en Red de Enfermedades Neurodegenerativas (CIBERNED), Madrid, Spain \\ ${ }^{\mathrm{c}}$ Neuro-oncology Unit, Instituto de Salud Carlos III-UFIEC, Majadahonda, Madrid, Spain \\ ${ }^{\mathrm{d}}$ Facultad de Ciencias Experimentales, Universidad Francisco de Vitoria, Pozuelo de Alarcón, Madrid, Spain
}

Accepted 9 May 2019

\begin{abstract}
Mitochondrial alterations and oxidative stress are common features of Alzheimer's disease brain and peripheral tissues. Moreover, mitochondrial recycling process by autophagy has been found altered in the sporadic form of the disease. However, the contribution of the main proteins involved in this pathology such as amyloid- $\beta$ protein precursor (A $\beta P P$ ) and tau needs to be achieved. With this aim, human unmodified fibroblasts were transduced with lentivectors encoding APP and Tau and treated with CCCP to study the mitophagy process. Both A $\beta P P$ and tau separately increased autophagy flux mainly by improving degradation phase. However, in the specific case of mitophagy, labeling of mitochondria by PINK1 and PARK2 to be degraded by autophagy seemed reduced, which correlates with the long-term accumulation of mitochondria. Nevertheless, the combination of tau and A $\beta P P$ was necessary to cause a mitophagy functional impairment reflected in the accumulation of depolarized mitochondria labeled by PINK1. The overexpression of Tau and APP recapitulates the mitophagy failure previously found in sporadic Alzheimer's disease.
\end{abstract}

Keywords: Alzheimer's disease, amyloid- $\beta$ protein precursor, mitochondria, mitophagy, tau

\section{INTRODUCTION}

Alzheimer's disease (AD) is the most common neurodegenerative disorder. The confirmed diagnosis of $\mathrm{AD}$ requires the presence of two major neuropathological lesions: accumulation of extracellular amyloid- $\beta$ (A $\beta)$ in senile plaques and intracellular

\footnotetext{
${ }^{1}$ Present address: Feil Family Brain and Mind Research Institute, Weill Cornell Medicine, New York, NY, USA.

${ }^{2}$ Present address: Departamento de Anatomía, Histología y Neurociencia, Facultad de Medicina, UAM, Madrid, Spain.

*Correspondence to: Jesús Avila and Vega García-Escudero, Centro de Biología, Molecular Severo Ochoa, Nicolás Cabrera, 1, Cantoblanco, 28049 Madrid, Spain. Tel.: +34 91196 4564/4592; Fax: +34 91196 4420; E-mails: javila@cbm.csic.es; v.garciaescudero@uam.es
}

microtubule-associated protein tau (MAPT/tau) in neurofibrillary tangles. $A \beta$ is generated via sequential proteolysis of amyloid- $\beta$ protein precursor $(\mathrm{A} \beta \mathrm{PP})$ [1], which is proteolyzed by $\beta$ - and $\gamma$-secretase to yield $A \beta$. Consecutive cleavages of $\beta$ - and $\gamma$ secretase generate peptides of 40 or 42 amino acids $\left(A \beta_{40}\right.$ or $\left.A \beta_{42}\right)$, which are released into the extracellular space [2]. $A \beta_{42}$ is more hydrophobic than $A \beta_{40}$, and tends to aggregate and fold in $\beta$-sheet structure, recruiting other extracellular proteins, which could result in the formation of senile plaques $[3,4]$. In $\mathrm{AD}$, tau is highly phosphorylated, and many of the tau phosphorylation sites are targeted by glycogen synthase kinase-3 (GSK-3) [5, 6]. Although altered proteolytic processing of $A \beta P P$ plays a central role in the production and accumulation of $A \beta$, failure of 
$\mathrm{A} \beta$ and tau clearance can contribute to the pathogenesis in sporadic $\mathrm{AD}$ [7]. It has been reported that $\mathrm{A} \beta$ accumulates within autophagic vacuoles in swollen dystrophic neurites in human AD brain, suggesting the involvement of autophagy in $\mathrm{AD}$ pathogenesis [8].

Autophagy is the major cellular pathway for degradation of long-lived and aggregated proteins, as well as cytoplasmic organelles $[9,10]$. In macroautophagy, cytoplasmic materials are engulfed by double membrane structures called autophagosomes, which subsequently, fuse with lysosomes to degrade their content [11]. Autophagy plays a fundamental role in neuronal function and is intensively involved in AD-related protein aggregation [8]. Indeed, it has been demonstrated to be the major degradational pathway following unfolded protein response activation in neuronal cells, an early event in AD brain [12].

It is well known that $A \beta$ and tau aggregation is associated with mitochondrial damage, oxidative stress, and cytoskeletal alteration of neurons in $\mathrm{AD}$ [13]. Mitophagy is the selective degradation of mitochondria by autophagy. We have recently demonstrated a defect in mitophagy process that causes the abnormal accumulation of mitochondria in peripheral and brain tissues from sporadic $\mathrm{AD}$ patients [14] which correlates with the increase of autophagic vesicles containing mitochondria previously found in pyramidal neurons in these patients $[15,16]$.

Although mitophagy alterations are well defined in $\mathrm{AD}$, the contribution of the two principal proteins involved in the disease, A $\beta P P$ and tau, to this failure needs to be clarified. Since, the majority of AD patients are sporadic, where no genetic mutations have been associated, we proposed to analyze the effect of wild type A $\beta P P$ and tau in mitophagy process. In fact, $\mathrm{AD}$ is a problem of accumulation of these proteins; therefore, we studied the effect of their overexpression. We have demonstrated that $A P P$ and $T a u$ overexpression generates an activation of autophagy flux, but a deficiency in the labeling of mitochondria to be recycled by autophagy. Our data revealed that the presence of tau aggravates the defect showed by APP overexpression leading to a compromised mitophagy process.

\section{MATERIALS AND METHODS}

\section{Primary cells and culture conditions}

Primary skin fibroblasts were obtained from Coriell Institute for Medical Research (AG11362;
New Jersey, USA). Human fibroblasts were cultured in Dulbecco's modified Eagle's medium (DMEM) supplemented with $10 \%(\mathrm{v} / \mathrm{v})$ heat-inactivated fetal bovine serum (FBS), $2 \mathrm{mM}$ glutamine, $10 \mathrm{U} / \mathrm{ml}$ penicillin, $10 \mu \mathrm{g} / \mathrm{ml}$ streptomycin, in $5 \% \mathrm{CO}_{2}$ in a humid incubator at $37^{\circ} \mathrm{C}$.

\section{Antibodies}

The primary antibodies used were: Tau5 (577801, Calbiochem), Tau404 (44758G, Life Technologies), Tau396 (44752G, Life Technologies), PHF1 [17] (a kind gift of Dr. P. Davies, Albert Einstein Coll., Bronx, NY, USA), Tau7.51 [18] (a kind gift of Dr. C. Wischik, MRC, Cambridge, UK), AT8 (MN1020, Innogenetics, Ghent, Belgium), AT100 (MN1060, Innogenetics), AT180 (MN1040, Innogenetics), $\beta$ Actin (A2228, Sigma-Aldrich), A $\beta P P$ (A8717, Sigma-Aldrich), $\beta$-Amyloid (803015, Biolegend), TOMM20 (sc-11415, Santa Cruz), LC3 (B7931, Sigma-Aldrich), p62 (610832, BD Bioscience), LAMP1 (611042, BD Bioscience), CatB (AB4064, Chemicon), GAPDH (ab8245, Abcam), PARK2 (sc32282, Santa Cruz), PINK1 (BC100-494, Novus), COX4 (sc-69359, Santa Cruz). The secondary antibodies for western blot studies were horseradish peroxidase-conjugated anti-rabbit or anti-mouse IgGs (P0448 and P0161, DAKO) and for immunofluorescence were anti-mouse or anti-rabbit IgGs alexa-488 or -555 labeled (Molecular Probes, Millipore).

\section{Western blot analysis}

The cells and tissue samples were homogenized in lysis buffer $(50 \mathrm{mM}$ pH $7.5 \mathrm{HCl}$-Tris, $300 \mathrm{mM}$ $\mathrm{NaCl}, 0.5 \%$ SDS and $1 \%$ Triton $\mathrm{X}-100$ ) and incubated $15 \mathrm{~min}$ at $95^{\circ} \mathrm{C}$. Protein concentration of the extracts was measured using the Dc protein assay kit (500-0111, Bio-Rad). Equal amounts of total protein extracts were resolved by SDS-PAGE and then transferred to nitrocellulose membranes (1040396, Watman Protean). Western blot and immunoreactive proteins were developed using an enhanced chemiluminescence detection kit (NEL105001EA, Perkin Elmer) following instructions of the supplier. Quantification was performed by densitometry of the obtained bands in each lane with respect to the correspondent housekeeping protein in each experiment (Quantity One software, Bio-Rad). Every data was normalized with respect to untreated control cells overexpressing GFP. 


\section{Autophagy flux analysis}

Fibroblasts were treated with CCCP $(20 \mu \mathrm{M})$ for $24 \mathrm{~h}$ followed by an additional treatment of PBS or $\mathrm{NH}_{4} \mathrm{Cl}(15 \mathrm{mM})$ for $6 \mathrm{~h}$ in the presence of CCCP. After the treatment, cells were lysed in western blot buffer and immunodetection of autophagy involved proteins was performed. Quantification of autophagic synthesis was represented as the ratio between the values of the cells treated with CCCP and $\mathrm{NH}_{4} \mathrm{Cl}$ with respect to the condition without CCCP but maintaining $\mathrm{NH}_{4} \mathrm{Cl}$ treatment. Quantification of autophagic degradation ratio was obtained by the relation between the values of the cells treated with $\mathrm{CCCP}$ and $\mathrm{NH}_{4} \mathrm{Cl}$ and the ones without $\mathrm{NH}_{4} \mathrm{Cl}$ but maintaining $\mathrm{CCCP}$ treatment according to autophagy standard guidelines $[19,20]$.

\section{Lentivirus production}

Pseudotyped lentivectors were produced using reagents and protocols from Didier Trono with the following modifications: 293T cells were transiently co-transfected with $5 \mu \mathrm{g}$ of the corresponding lentivector plasmid, $5 \mu \mathrm{g}$ of the packaging plasmid pCMVdR8.74 (Addgene plasmid 22036), and $2 \mu \mathrm{g}$ of the VSV $\mathrm{G}$ envelope protein plasmid pMD2G (Addgene plasmid 12259) using Lipofectamine and Plus reagents following instructions of the supplier (18324 and 11514, respectively, Life Technologies). The lentivectors used encode wild type human APP (provided by Dr. Jason Jon Fritz, Emory University School of Medicine, Atlanta, GA) [21], or the longest wild type isoform of MAPT/Tau in human brain harboring four repeats and two $\mathrm{N}$-terminal inserts followed by GFP linked by an IRES (kindly ceded by Prof. Kenneth S. Kosik, UC Santa Barbara, CA). As a control we used a lentivector encoding $E$ GFP, pRRLSIN.cPPT.PGK-GFP.WPRE (Addgene plasmid 12252).

\section{Mitochondrial potential measurement}

Cells were treated with $20 \mu \mathrm{M}$ carbonyl cyanide m-chlorophenylhydrazone (CCCP; C2759, Sigma) for $7 \mathrm{~h}$ and, in the reversible condition, CCCP was removed and the medium was replaced for DMEM $10 \%$ FBS during the last $60 \mathrm{~min}$. Then, fibroblasts were incubated with 50 nM DiOC6(3) (D273, Molecular Probes) for $30 \mathrm{~min}$ at $37^{\circ} \mathrm{C}$ and analyzed by flow cytometry (FACSCalibur, BD Biosciences, San Jose, CA). Mitochondrial membrane potential for each condition was represented as the fluorescence intensity in arbitrary units.

\section{Lysosomal function study}

Cells were treated with $100 \mathrm{nM}$ bafilomycin A1 (B1793, Sigma) for $2 \mathrm{~h}$ or remain untreated. After that, cells were collected and treated with 500 nM LysoTracker Red DND-99 (L-7528, Molecular Probes, Life Technologies) for $20 \mathrm{~min}$. Then, cells were analyzed by flow cytometry (FACSCalibur, BD Biosciences, San Jose, CA). Relative LysoTracker level represents the ratio of the FL-2 fluorescence intensity of the untreated condition and the bafilomycin treated one.

\section{Immunocytochemistry}

Fibroblasts and differentiated neurons were grown on sterile glass coverslips, treated as described for each experiment, followed by washing with PBS and fixing with $4 \%$ paraformaldehyde in PBS for 30 min at room temperature. After blocking with PBS containing $1 \% \mathrm{BSA}$, permeabilizing with $0.1 \%$ Triton X-100 and Glycine $1 \mathrm{M}$ for 30 min, cells were washed with PBS and stained by indirect immunofluorescence using the antibodies described before. When indicated, phalloidin (A-12379, Life Technologies) was added at 1:200 during the second antibody incubation. Samples were mounted with Prolong Gold Antifade (P-36930, Life Technologies) and randomly chosen field images were obtained in an Invert Confocal LSM510 (Zeiss, Oberkochen, Germany) fluorescence microscope.

\section{Colocalization assay}

Colocalization analysis was performed with ImageJ software (Bethesda, MD) and every cell was manually delimited according to phalloidin staining. The background of different channels was edited with Subtract Background tool with a rolling ball radius of 30 pixels; and by a threshold intensity, binary images were obtained. The logical operation AND of the Image Calculator tool was used to generate an image harboring only overlapping structures of both channels. Colocalization measurement was obtained by quantifying the area occupied by the overlapping elements per cell. At least 200 cells were measured for each cell line. 


\section{Statistical analysis}

Graphs represent means and standard deviations of the values obtained from experimental triplicates. Statistical comparison of the data sets was performed by the Student's $t$ test. Two-way ANOVA test was performed to examine the differences between experimental factors and their interaction. A post-hoc Bonferroni test was used when more than two experimental groups were compared. The differences are given with their corresponding statistical significance or $p$ value, which is the probability that the difference occurred merely by chance under the null hypothesis.

\section{RESULTS}

\section{APP overexpression increased autophagy degradation phase}

To study the autophagy process, cells were infected with a lentivector encoding wild type (WT) APP compared to cells transduced by a lentivector encoding enhanced-GFP as a protein overexpression control (Supplementary Figure 1). First, we analyzed the $\mathrm{A} \beta$ production in our cell model. As it was previously described [22], the intracellular overexpression of WT APP promoted $\mathrm{A} \beta$ oligomer accumulation respect to the levels in control cells (Supplementary Fig. 2A, B). Then, cells were treated for $24 \mathrm{~h}$ with the mitochondrial respiratory chain uncoupler carbonyl cyanide m-chlorophenylhydrazone (CCCP), which triggers loss of mitochondrial membrane potential. This was followed by an additional treatment of $\mathrm{NH}_{4} \mathrm{Cl}$ for $6 \mathrm{~h}$ in the presence of CCCP to block the degradation phase of autophagy. APP-expressing cells showed a decreased amount of autophagic vesicles (AV) revealed by the lower levels of LC3II protein as well as LC3II/LC3I ratio under basal conditions (Fig. 1A-C). These results were accompanied by unchanged $\mathrm{AV}$ synthesis along with a significant increase in their degradation (Fig. 1D, E). This may explain the diminished AV content observed. Proper lysosomal function is essential for degrading proteins, organelles and large protein aggregates or inclusions by the autophagic pathway. Therefore, we examined levels of lysosomal-associated membrane protein 1 (LAMP1), which was markedly enhanced in $A P P$-expressing cells (Fig. 1F, G). It is well known that the lysosomal cysteine protease Cathepsin $\mathrm{B}$ has been associated with amyloid plaques and increased $\mathrm{A} \beta$ production [23, 24]; thus, levels of the active form of this protease were studied and revealed a considerable increase in $A P P$-expressing cells (Fig. 1F, G). These results together suggested an enhanced degradation phase of autophagy correlating with a higher lysosomal content causing reduced AV levels.

\section{Imbalance of mitophagy-related proteins after APP overexpression}

The removal of damaged mitochondria is critical for maintaining proper cellular functions [25]. This process is mediated by autophagy mechanisms and it is named mitophagy. PARK2 is a ubiquitin ligase involved in the labeling of defective mitochondria to be targeted for autophagic recycling. It has been demonstrated that within $1 \mathrm{~h}$ of CCCP addition, endogenous PARK2 was recruited to mitochondria in the majority of cells [26]. We could also confirm that in our fibroblast model mitochondrial localization of both PINK1 and PARK2 proteins was increased after $1 \mathrm{~h}$ of CCCP treatment (Fig. 2A). Therefore, to study mitophagy, we quantify mitochondrial localization of PARK2 in such conditions. Immunofluorescence study displayed a decreased mitochondrial localization of PARK2 in APP-expressing cells suggesting a defective labeling of damaged mitochondria to be recycled by mitophagy (Fig. 2B, C). Conversely, PARK2 levels study by western blot revealed substantial accumulation of this protein in $A P P$-expressing cells (Fig. 2D). It has been described that PARK2 is recruited to the mitochondria by a PTEN-induced putative kinase 1 (PINK1)-dependent mechanism for mitochondrial turnover by the autophagy system [27]. After mitochondrial damage, PINK1 is stabilized in the mitochondrial membrane as a full length (FL) isoform which recruits PARK2 [28]. Immunofluorescence analysis of the stabilization of PINK1 in the mitochondria after CCCP challenge for $1 \mathrm{~h}$ showed a diminished PINK1 content at this subcellular localization suggesting a defective labeling of damaged mitochondria for mitophagy degradation (Fig. 2E, F). High levels of PARK2 and low PARK2 and PINK1 mitochondrial targeting may be explained by increased autophagy flux that rapidly degrade damaged mitochondria. Then, we studied mitophagy process long-term after CCCP treatment for $24 \mathrm{~h}$. In such conditions, we could observe the augmented levels of FL-PINK1 in APP overexpressing cells with respect to control cells, suggesting the long-term accumulation of damaged mitochondria labeled by FL-PINK1 (Fig. 2G). On the other hand, it has been demonstrated PINK1 may also play 


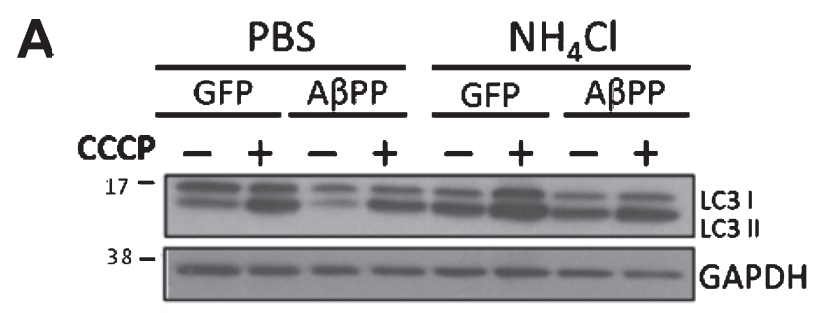

B

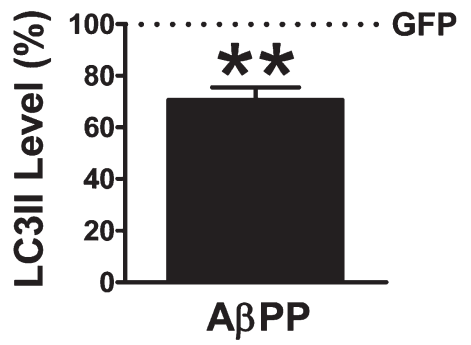

D

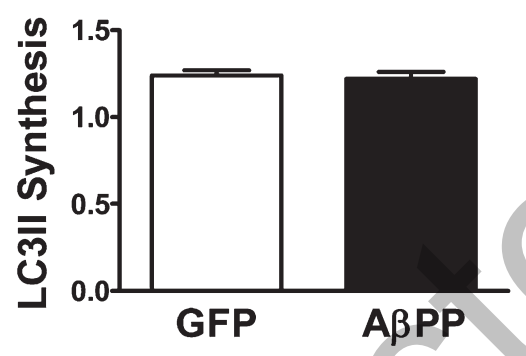

C

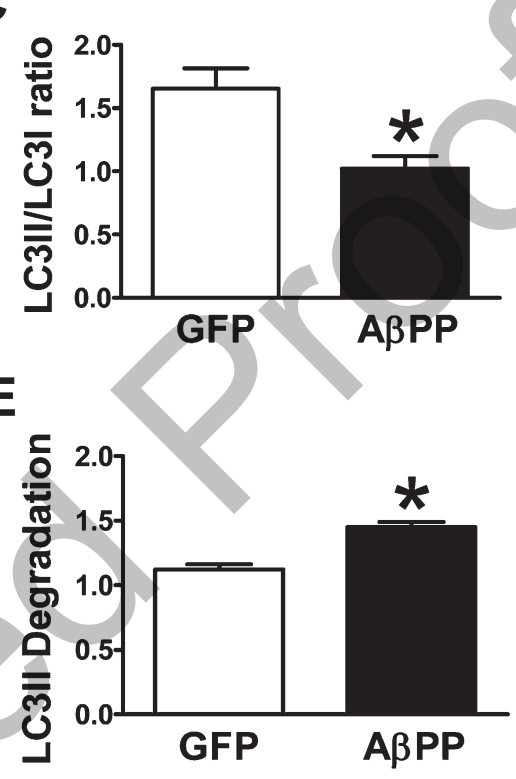

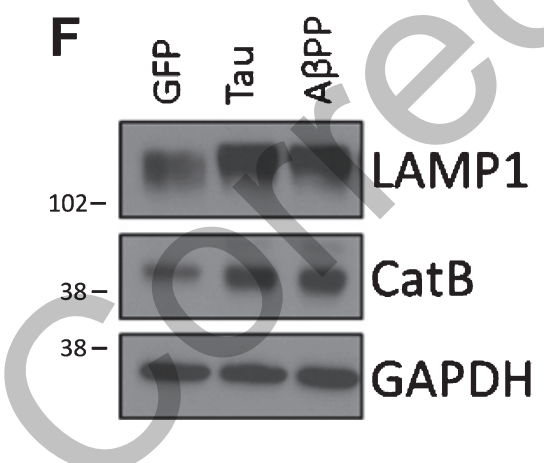

$\mathbf{G}$

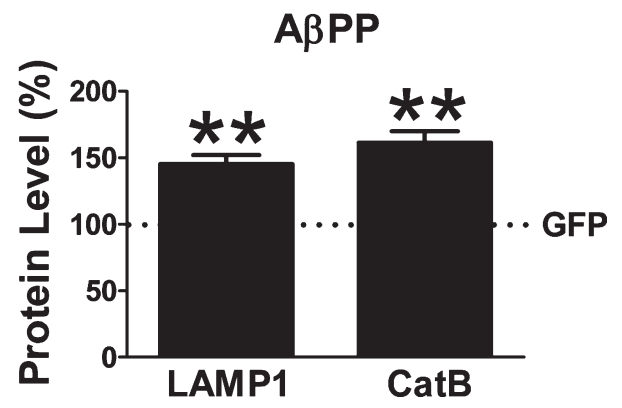

Fig. 1. APP overexpression enhanced degradation phase of autophagy. A) Representative western blot of LC3 expression for the study of autophagy flux as explained in Material and Methods. B) Quantification of LC3II levels in APP-expressing cells with respect to GFPexpressing cells under basal conditions. C) Quantification of LC3II/LC3I ratio in APP-expressing cells with respect to GFP under basal conditions. D, E) Quantification of LC3II synthesis (D) and degradation (E) ratios as described in Material and Methods. F, G) Representative western blot and quantification of LAMP1 and Cathepsin B levels after APP overexpression with respect to GFP. ( $n=3$ independent experiments; $\left.{ }^{*} p<0.05,{ }^{* *} p<0.01\right)$.

an inhibitory role in mitophagy process [29]. The main cleaved product of PINK1 ( $\Delta 1$ fragment) is able to physically bind PARK2 in the cytosol inhibiting its translocation to the mitochondria, therefore, impairing the elimination of damaged mitochondria. Accordingly, APP-expressing cells exhibited elevated levels of $\triangle 1$-PINK1 after $24 \mathrm{~h}$ of CCCP treatment, suggesting that this inhibitory mechanism may contribute to diminish PARK2 mitochondrial levels while whole cell levels were increased causing the accumulation of damaged mitochondria labeled by FL-PINK1 (Fig. 2G). Accordingly, we observed 


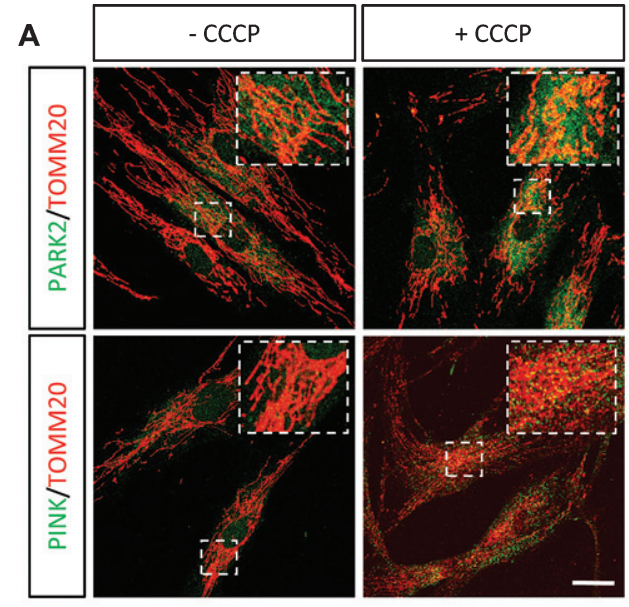

C
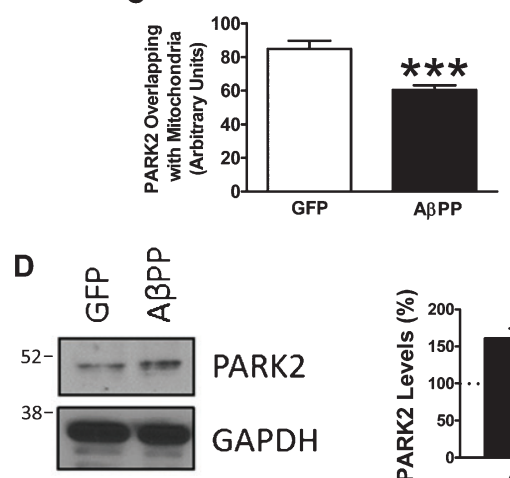

$\mathbf{F}$
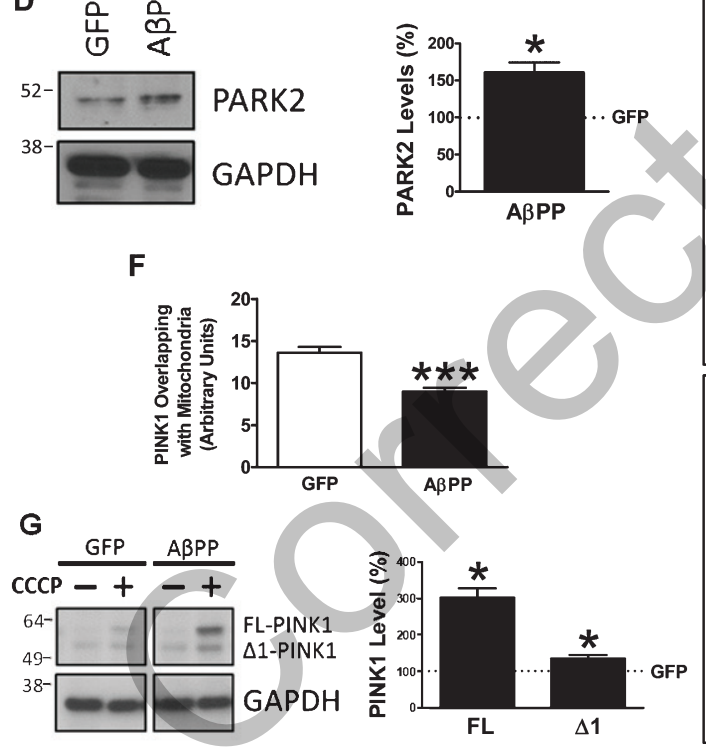
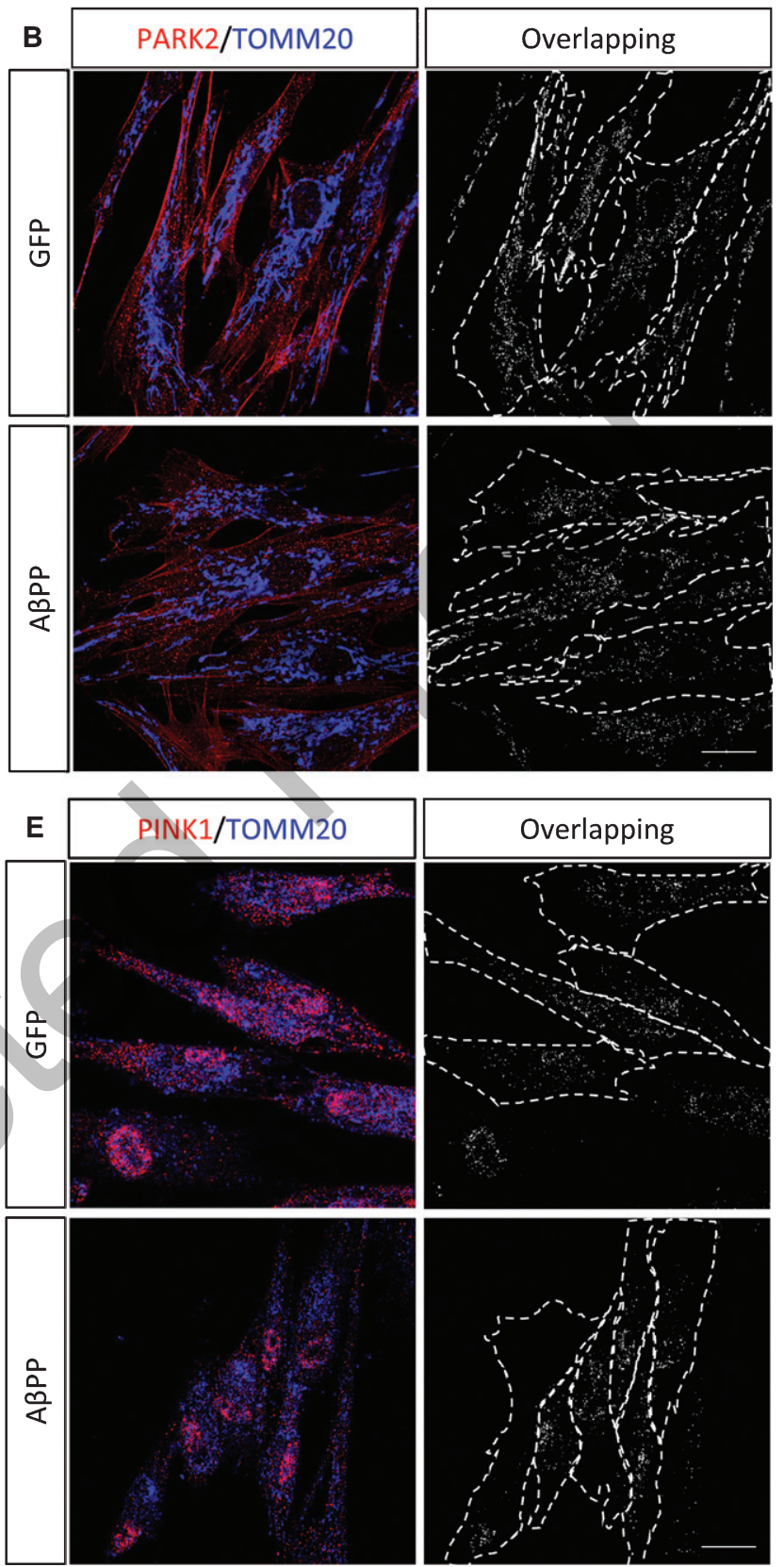

Fig. 2. Imbalance pattern of PARK2 and PINK1 in APP-expressing cells. A) Representative confocal microscopy immunofluorescence images of control fibroblasts treated with vehicle (control) or CCCP for $1 \mathrm{~h}$ showing TOMM20 in red and PARK2 (upper panel) and PINK1 (bottom panel). Inset: magnification of the boxed area. B) Representative confocal microscopy immunofluorescence images of fibroblasts overexpressing GFP or APP showing PARK2 in red and TOMM20 as a mitochondrial constitutive marker in blue in the same cells treated with CCCP $(20 \mu \mathrm{M})$ for $1 \mathrm{~h}$. On the right, binary images representing the colocalization of both labels and dotted line delimits cytoplasm of each cell according to phalloidin staining (not shown). C) Quantification of the colocalization between PARK2 and TOMM20 expressed as area occupied by the overlapping elements per cell. D) Representative western blot and quantification of PARK2 basal levels. E) Representative confocal microscopy immunofluorescence images showing PINK1 in red and TOMM20 in blue of $A P P$ - or $G F P$-expressing fibroblasts treated with CCCP $(20 \mu \mathrm{M})$ for $1 \mathrm{~h}$. F) Quantification of the colocalization between PINK1 and TOMM20 expressed as area occupied by the overlapping elements per cell. G) Representative western blot of the expression of PINK1 in fibroblasts overexpressing GFP or APP treated with CCCP $(20 \mu \mathrm{M})$ for $24 \mathrm{~h}$. Quantification of the levels of FL-PINK1 and $\triangle 1$-PINK1 isoform in $A P P$-expressing cells with respect to GFP in the presence of CCCP. Scale bar: $40 \mu \mathrm{m}$. ( $n=3$ independent experiments; $\left.{ }^{*} p<0.05,{ }^{* * *} p<0.001\right)$. 

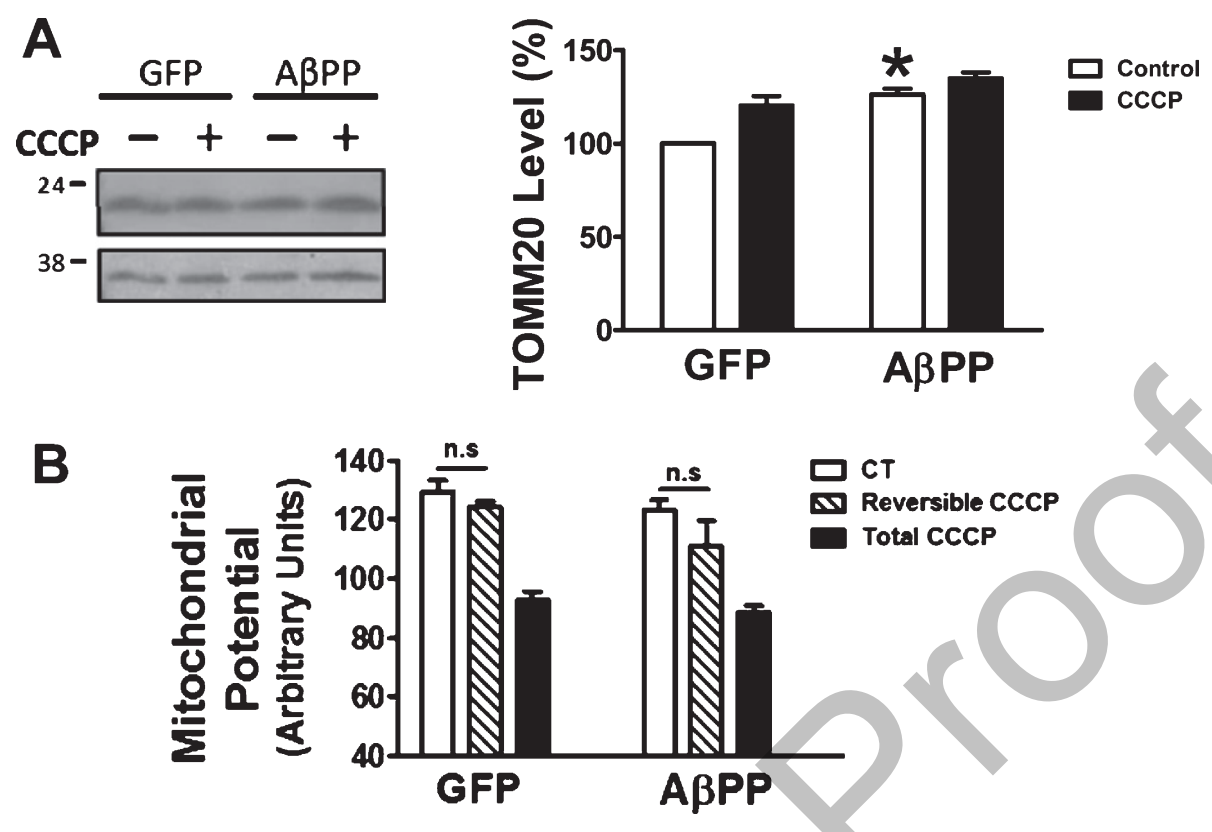

Fig. 3. APP overexpression caused mitochondrial accumulation. A) Representative western blot of TOMM20 protein levels in the absence or presence of CCCP $(20 \mu \mathrm{M})$ in fibroblasts overexpressing $G F P$ or $A P P$ and quantification of the levels under basal conditions. B) Mitochondrial membrane potential of the same fibroblasts reversibly untreated $(\mathrm{CT})$ or treated with CCCP $(20 \mu \mathrm{M})$ for $6 \mathrm{~h}$ and then allowed to recover for $1 \mathrm{~h}$ (reversible CCCP), or treated for $7 \mathrm{~h}$ with CCCP (total CCCP). $(n=4$ in A and $n=3$ in B independent experiments; $n . s$ : not significant, $\left.{ }^{*} p<0.05\right)$.

that mitochondrial constitutive protein TOMM20 levels were slightly higher in $A P P$ cells in respect to the controls (Fig. 3A). The increase of mitochondria per cell [30] and, even, increased levels of TOMM20 by western blot was previously related with the impairment of mitophagy. To test whether there was a mitochondria recycling failure, we analyzed the recovery of mitochondrial function represented by the mitochondrial membrane potential $(\Delta \Psi \mathrm{m})$ after a reversible treatment with CCCP. The overexpression of $A P P$ did not significantly alter $\triangle \Psi \mathrm{m}$ and depolarization took place similarly in both $G F P$ and $A P P$-expressing cells (Fig. 3B). After CCCP removal, $\triangle \Psi \mathrm{m}$ recovery took place in both $G F P$ and $A P P$-expressing cells although levels reached by $A P P$-expressing cells were to some extent lower (Fig. 3B). The short-term diminished targeting of PARK2 and PINK1 to mitochondria may explained by the observed increased autophagy flux; however, the long-term accumulation of FL-PINK1 together with increased mitochondrial content suggests a slightly compromised mitophagy process. This may be caused by insufficient labelling of depolarized mitochondria by PINK1 and PARK2. Additionally, the enhanced amount of $\triangle 1$-PINK1 may point out a further inhibitory mechanism over PARK2 translo- cation to mitochondria that may contribute to the deficient labeling of this damage organelle and that would explain the increased levels of this protein.

\section{Synergistic effect of Tau and APP causes an increased autophagy flux}

As MAPT/Tau is mainly expressed in neurons while $A P P$ is more widely expressed in the organism, we wanted to evaluate the contribution of Tau over the previously described effect of APP overexpression. Therefore, we studied the effect of tau and $\mathrm{A} \beta \mathrm{PP}$ in the process of autophagy by the overexpression of both proteins at the same time in a human line of skin fibroblasts by lentiviral vectors (Supplementary Figure 1). As in previous experiments, GFP overexpression was used as control and data were also compared with the overexpression of MAPT/Tau alone. As expected, Tau overexpression did not significantly affect the $A \beta$ oligomers production either alone or in combination with $A P P$ overexpression (Supplementary Figure 2A, B). As tau phosphorylation plays a fundamental role in AD pathology, we first characterized tau phosphorylation pattern after $A P P$ or/and Tau overexpression. We could observe that the overexpression of both Tau and APP/Tau 
exhibited phosphorylation in some of the typical ADrelated epitopes such as PHF1 phosphorylation sites Ser396/Ser404 and AT8 sites Ser202/Thr205 (Supplementary Figure 2C). On the contrary, part of the epitopes remained dephosphorylated as shown by Tau 1 antibody label (Dephosho Ser195, 198, 199, and 202) and negative staining for phospho-Thr231 (AT180) as well as phospho-Thr212 and phosphoSer214 (AT100). As expected, the co-expression of $T a u$ and APP did not affect the phosphorylation patterns with respect to the expression of Tau alone. These results indicate that the overexpression of Tau in vitro can induce its phosphorylation by the cell similar to what is found in AD brain.

The cells were treated for $24 \mathrm{~h}$ with CCCP followed by an additional treatment of $\mathrm{NH}_{4} \mathrm{Cl}$ for $6 \mathrm{~h}$ in the presence of CCCP. First, we showed that Tau overexpression decreased autophagic vesicles formation (LC3II/LC3I ratio) and tended to increase degradation ratio (Fig. 4A-E), whereas Tau/APP overexpression caused a considerable reduction of AVs content exhibited by lower levels of LC3II and LC3II/LC3I ratio under basal conditions that correlated with the activation of autophagy flux (Fig. 5A-C). This activation was indicated by the markedly elevated synthesis of AVs besides the significant increase of LC3II degradation when Tau and $A P P$ are overexpressed (Fig. 5D, E). Under the same conditions, we examined levels of p62, an adaptor protein that recognizes substrates to be recycled by autophagy being itself degraded in this process [31]. Western blot revealed diminished p62 levels in cells that overexpressed Tau and APP under basal conditions (Fig. 5F, G). Similar to previous situation, the p62 accumulation and degradation rates increased promoting the turnover of this protein and preventing their accumulation corroborating in an increased autophagy flux (Fig. 5H, I). Additionally, we could observe an increased lysosomal content either in Tau (Fig. 4F-H) or TaulAPP (Fig. 5J-L) overexpression indicated by the augmented levels of LAMP1 and active Cathepsin B. To ascertain if these lysosomes were functional, we studied their $\mathrm{pH}$ by LysoTracker assay finding that Tau/APP-expressing cells exhibited a higher capacity to retain LysoTracker probe suggesting an increased amount of lysosomes (Fig. 5M). All this data together indicate that although Tau overexpression induce a slight autophagy flux increase reflected by modestly augmented lysosomal content and LC3II degradation ratio, the combination of Tau and APP overexpression boosted autophagy flux by increasing synthesis and degradation what was accompanied by a marked increase in the lysosomal content.

\section{Tau and APP overexpression induces mitophagy impairment}

Taking into account that both tau and A $\beta P P$ contribute to mitochondrial pathogenesis in AD [13], we wanted to evaluate whether their overexpression could promote a defect in the mitochondrial recycling process. Accordingly, as it was previously seen for APP, Tau overexpression showed a diminished recruitment of PARK2 to the mitochondria after CCCP treatment for $1 \mathrm{~h}$ (Fig. 6A, B), which was even more drastic in APP and Tau combination (Fig. 7A, B). This situation may reflect an activation of detoxification system [32]. Additionally, when we studied whole cell levels of PARK2, we could observe that the overexpression of Tau caused an increase of PARK2 basal levels (Fig. 6C). Moreover, the combination of Tau and APP caused a synergic accumulation of PARK2 basal levels (Fig. 7C). Strikingly, Tau overexpression showed a diminish amount of PINK1 stabilized in mitochondria suggesting an enhanced recycling of damage mitochondria or a defect in mitophagy labeling (Fig. 6D, E), as was the case for $A P P$ (Fig. 2E, F). On the contrary, the combination of Tau and APP showed a dramatically increased stabilization of PINK1 in the mitochondria (Fig. 7D, E). Additionally, western blot analysis displayed that both FL-PINK1 and $\triangle 1$-PINK1 were significantly increased in Tau and Tau/APP-expressing cells after a mitochondrial insult for $24 \mathrm{~h}$ (Figs. $6 \mathrm{~F}$ and $7 \mathrm{~F}$, respectively). The increased levels of $\triangle 1$-PINK1 may sequester PARK2 in the cytosol impairing its targeting to mitochondria. The long-term accumulation of FL-PINK1 indicate that $\mathrm{A} \beta \mathrm{PP} /$ tau combination caused a mitophagy impairment similar to what it was taken place for A $\beta P P$ or tau separately. However, the main difference came when we analyze subcellular localization of PINK1 after CCCP challenge for $1 \mathrm{~h}$. This data indicates that upon APP and Tau overexpression, there was a correct stabilization of PINK1 in depolarized mitochondria. However, PARK2 was not properly targeted to this organelle suggesting a deficient crosstalk between these two proteins, which causes the accumulation of depolarized mitochondria. This data substantially correlates with the elevated TOMM20 levels shown in Tau/APP-expressing cells, which were more significantly increased than $A P P$ or Tau separately, suggesting the accumulation of 

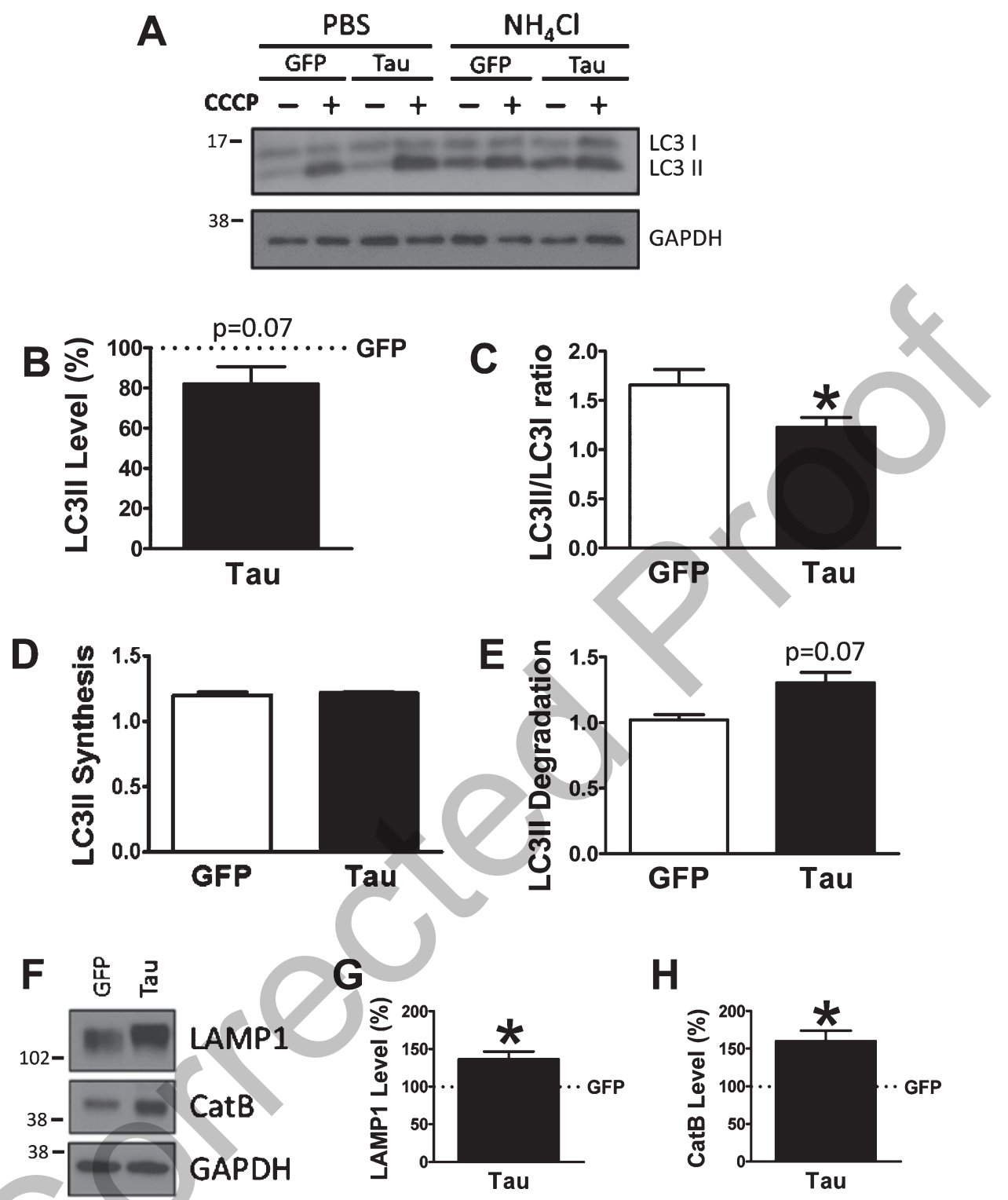

Fig. 4. Tau overexpression induced clearance of autophagic vesicles by enhanced lysosomal activity. A) Representative western blot of LC3 expression for the study of autophagy flux as explained in Methods in fibroblasts overexpressing GFP and Tau. B) Quantification of LC3II levels in Tau-expressing cells with respect to GFP-expressing cells under basal conditions. C) Quantification of LC3II/LC3I ratio in Tau-expressing cells with respect to GFP under basal conditions. D) Quantification of LC3II synthesis ratio as described in Methods. E) Quantification of LC3II degradation ratio as described in Methods. F-H) Representative western blot and quantification of LAMP1 and Catepsin B proteins under Tau overexpression with respect to GFP. ( $n=4$ in A-E and $n=3$ in F-H independent experiments; $p$ values are shown in the corresponding graphs, $\left.{ }^{*} p<0.05\right)$.

dysfunctional mitochondria (Fig. 8A; Supplementary Figure 3A). Similar results were obtained using a matrix mitochondrial protein such as cytochrome c oxidase complex IV (COX4, Supplementary Figure 3B). To study if this deficient crosstalk would be translated into a functional mitophagy impairment, we studied $\Delta \Psi \mathrm{m}$ recovery. It has been previously demonstrated that $6 \mathrm{~h}$ CCCP challenge leads to full- length PINK1 stabilization and PARK2 recruitment to damaged mitochondria and ubiquitination of mitochondrial PARK2 targets that are recognized by p62, to the their subsequent target into autophagosomes and further elimination [33]. Moreover, if CCCP is removed from the media, dysfunctional mitochondria labeled by full-length PINK1 are eliminated in $1 \mathrm{~h}$ [34] in a PARK2-dependent mitophagy process [35]. 


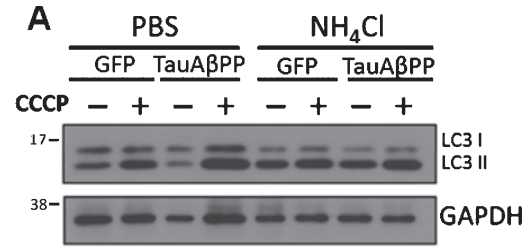

B

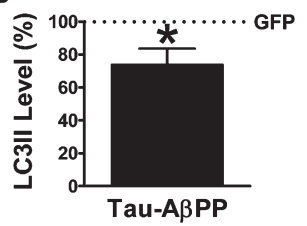

D
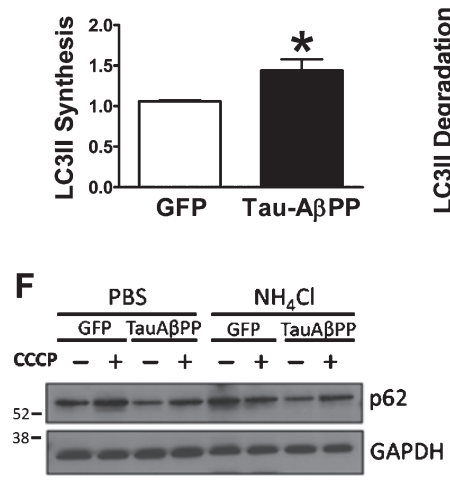

C

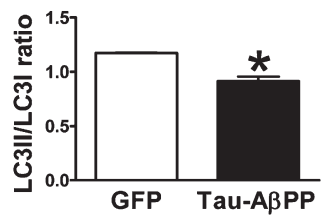

E

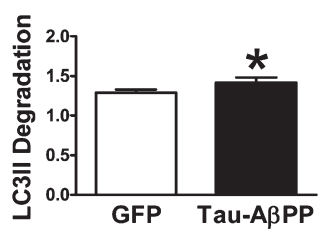

G

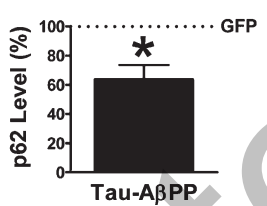

H
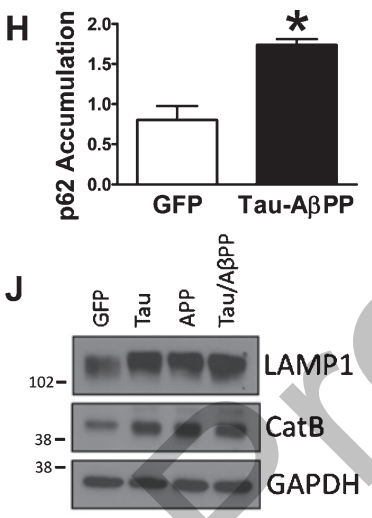

L

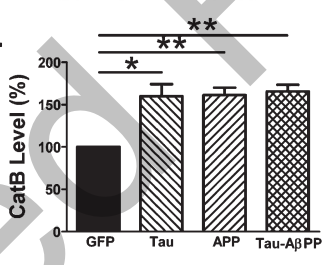

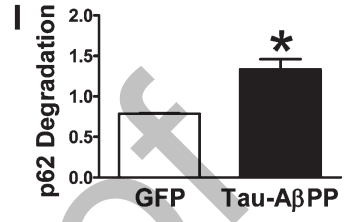
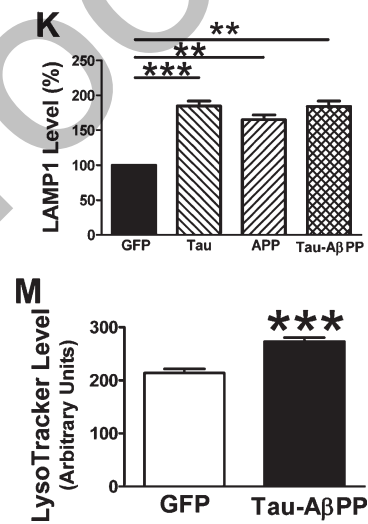

Fig. 5. Increased autophagy flux and lysosomal activity caused by APP and Tau overexpression. A) Representative western blot of LC3 expression for the study of autophagy flux as explained in Material and Methods. B) Quantification of LC3II levels in Tau/APP-expressing cells with respect to GFP-expressing cells under basal conditions. C) Quantification of LC3II/LC3I ratio in GFP- or Tau/APP-expressing cells under basal conditions. D, E) Quantification of LC3II synthesis (D) and degradation (E) ratio as described in Material and Methods. F, G) Representative western blot of p62 expression after the treatment as in (A) and quantification of basal levels. H) Quantification of p62 synthesis ratio as described for (D). I) Quantification of p62 degradation ratio as defined in (E). J-L) Representative western blot and quantification of LAMP1 (K) and Catepsin B (L) levels in all conditions. M) Quantification of the amount of lysosomes per cell in GFP-or Tau/APP-expressing cells. ( $n=4$ in A-E and $n=3$ in F-M independent experiments; $\left.{ }^{*} p<0.05,{ }^{* *} p<0.01,{ }^{* * *} p<0.001\right)$.

Using similar reversible CCCP challenge, Tau/APPexpressing cells showed significantly diminished $\triangle \Psi \mathrm{m}$ recovery with respect to $G F P$-cells suggesting that depolarized mitochondria were not properly eliminated in this timeframe by mitophagy (Fig. 8B). These results together indicate that the combination of Tau and APP overexpression disrupt the mitophagy process causing the accumulation of dysfunctional low-potential mitochondria labeled by PINK1.

\section{DISCUSSION}

Extensive literature exists supporting the role of mitochondrial dysfunction and oxidative damage in the early $\mathrm{AD}$ pathogenesis $[13,36,37]$. There are strong indications that oxidative stress occurs prior to the onset of symptoms in $\mathrm{AD}$ and, moreover, oxidative damage is found not only in the vulnerable regions of affected brain in the disease [36, 38] but also in peripheral cells [39]. On the other hand, autophagy plays a fundamental role in neuronal function and is intensively involved in AD-related protein aggregation [8]. Moreover, the mitochondrial recycling by autophagy has been recently demonstrated to be altered in sporadic AD [14]; however, the contribution of A $\beta P P$ and tau to mitophagy dysfunction was unexplored. In this regard, several works have pointed out that $A \beta$ and tau may interfere with cell functions that are fundamental for mitophagy process. Segregation of damaged mitochondria to be recycled by mitophagy depends on fission and fusion events that are altered in AD [40]. It has been described that abnormal interaction of $A \beta$ monomers and oligomers as well as hyperphosphorylated tau with the mitochondrial fission protein DLP1 

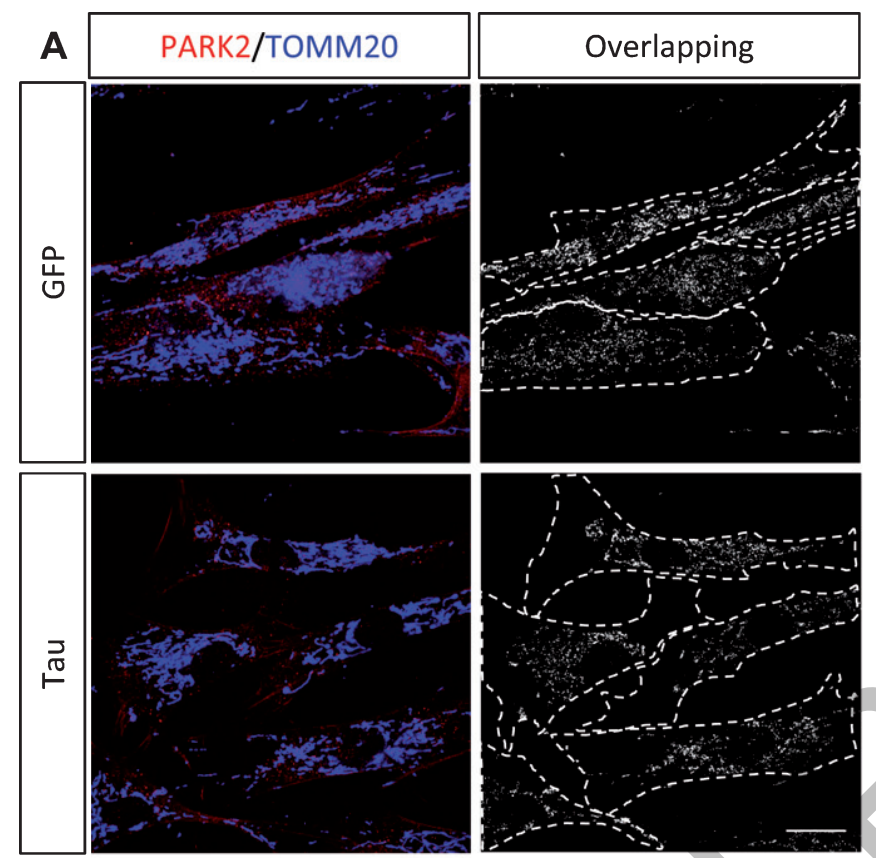

B
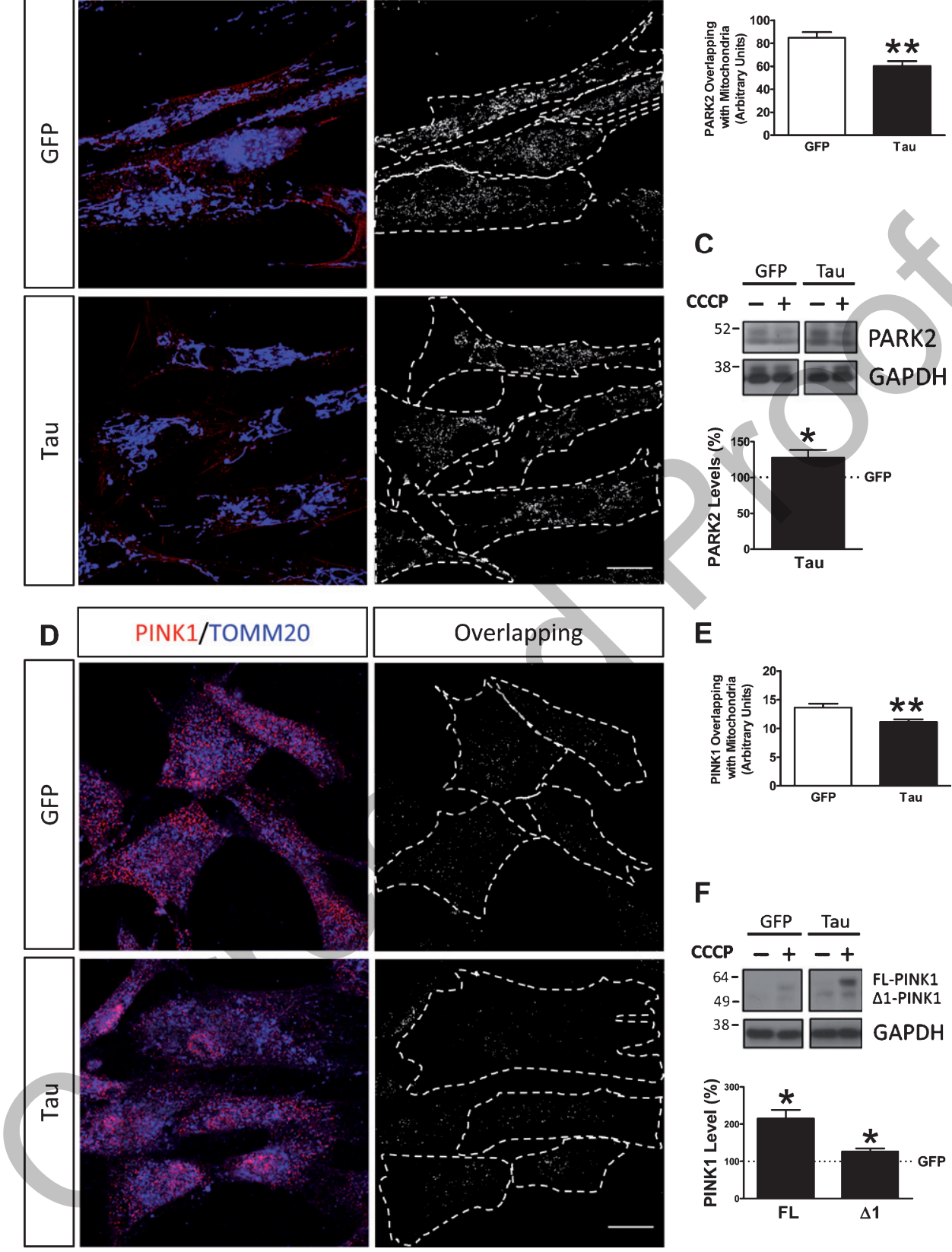

Fig. 6. Imbalance pattern of PARK2 and PINK1 in Tau-expressing cells. A) Representative confocal microscopy immunofluorescence images showing PARK2 in red and TOMM20 as a mitochondrial constitutive marker in blue in the fibroblasts overexpressing GFP or Tau treated with CCCP $(20 \mu \mathrm{M})$ for $1 \mathrm{~h}$. On the right, binary image representing the colocalization of both labels and dotted line delimits cytoplasm of each cell according to phalloidin staining (not shown). B) Quantification of the colocalization between PARK2 and TOMM20 expressed as area occupied by the overlapping elements per cell. C) Representative western blot in the absence or presence of CCCP for $24 \mathrm{~h}$ and quantification of PARK2 in fibroblasts overexpressing GFP or Tau and quantification of the levels under basal conditions. D) Representative confocal microscopy immunofluorescence images showing PINK1 in red and TOMM20 in blue of GPF- or Tau-expressing fibroblasts treated with CCCP $(20 \mu \mathrm{M})$ for $1 \mathrm{~h}$. On the right, binary image representing the colocalization of both labels and dotted line delimits cytoplasm of each cell according to phalloidin staining (not shown). E) Quantification of the colocalization between PINK1 and TOMM20 expressed as area occupied by the overlapping elements per cell. F) Representative western blot of the expression of PINK1 in fibroblasts overexpressing $G F P$ or $A P P$ treated with CCCP $(20 \mu \mathrm{M})$ for $24 \mathrm{~h}$. Quantification of the levels of FL-PINK1 and $\Delta 1$-PINK1 isoform in Tau-expressing cells with respect to GFP in the presence of CCCP. Scale bar: $40 \mu \mathrm{m}$. $\left(n=3\right.$ independent experiments; $\left.{ }^{*} p<0.05,{ }^{* *} p<0.01\right)$. 

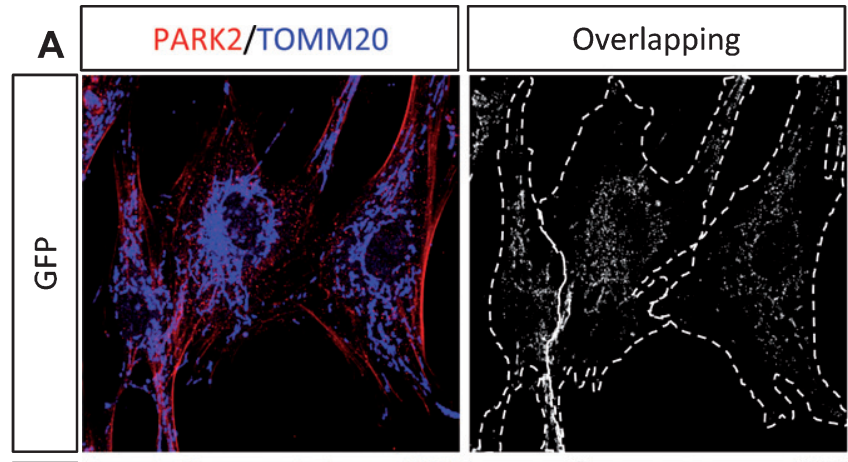

B
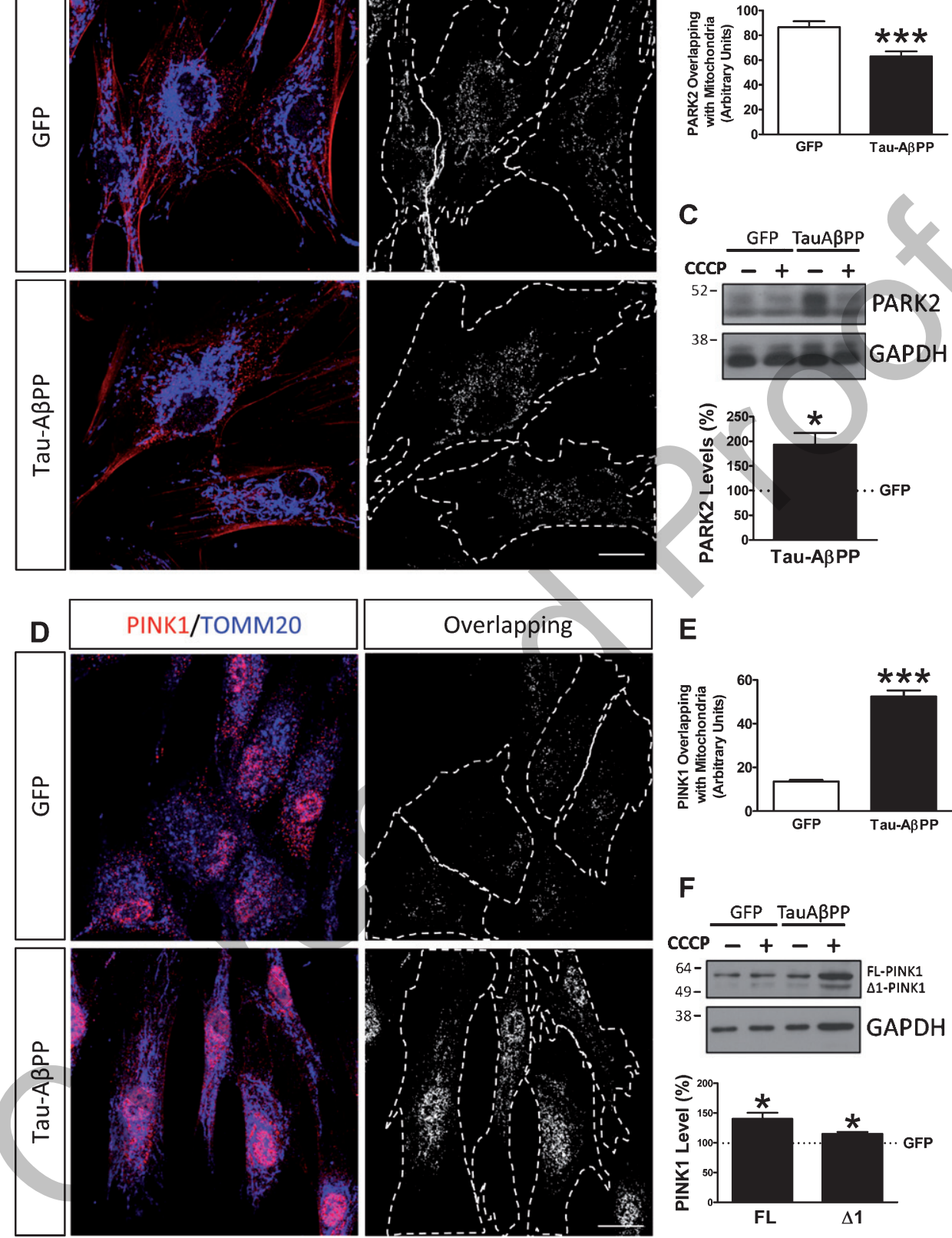

Fig. 7. Imbalance pattern of PARK2 and PINK1 in Tau/APP-expressing cells. A) Representative confocal microscopy immunofluorescence images showing PARK2 in red and TOMM20 as a mitochondria constitutive marker in blue of GFP- or Tau/APP-expressing fibroblasts treated with $\mathrm{CCCP}(20 \mu \mathrm{M})$ for $1 \mathrm{~h}$. On the right, binary image representing the colocalization of both labels and dotted line delimits cytoplasm of each cell according to phalloidin label (not shown). B) Quantification of the colocalization between PARK2 and TOMM20 expressed as area occupied by the overlapping elements per cell. C) Representative western blot of PARK2 in fibroblasts overexpressing GFP or Tau and $A P P$ in the absence or presence of CCCP $(20 \mu \mathrm{M})$ and quantification of the levels under basal conditions. D, E) Representative confocal microscopy immunofluorescence images showing PINK1 in red and TOMM20 in blue of GFP- and Tau/APP-expressing fibroblasts treated with CCCP $(20 \mu \mathrm{M})$ for $1 \mathrm{~h}$. On the right, binary image representing the colocalization of both labels and dotted line delimits cytoplasm of each cell according to phalloidin label (not shown) and quantification of the data (E). F) Representative western blot of the expression of PINK1 in fibroblasts overexpressing GFP or Tau/APP treated with CCCP $(20 \mu \mathrm{M})$ for $24 \mathrm{~h}$. Quantification of the levels of FL-PINK1 and $\triangle 1$-PINK1 isoforms in Tau/APP-expressing cells with respect to GFP in the presence of CCCP. Scale bar: $40 \mu \mathrm{m}$. $(n=3$ independent experiments; $\left.{ }^{*} p<0.05,{ }^{* * *} p<0.001\right)$. 

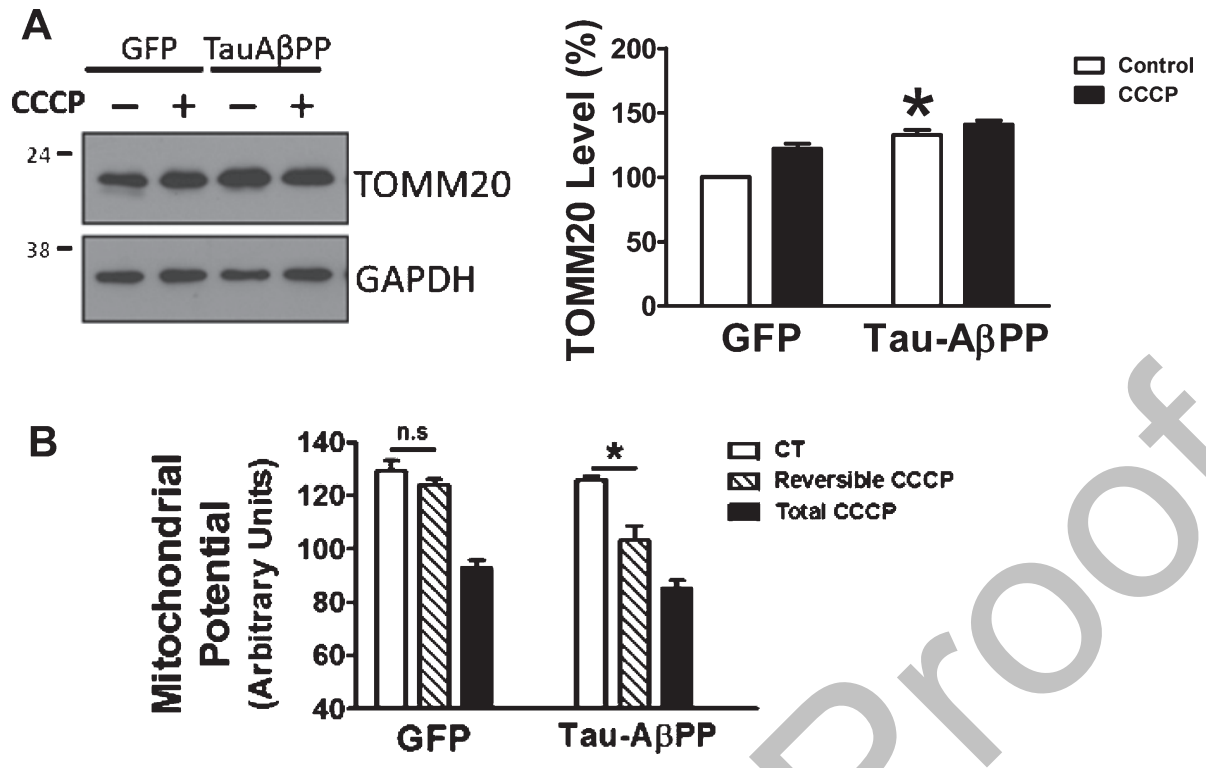

Fig. 8. Increased mitochondrial content in Tau/APP-expressing cells. A) Representative western blot of TOMM20 protein levels in fibroblasts overexpressing GFP or APP/Tau in the absence or presence of CCCP $(20 \mu \mathrm{M})$ and quantification of the levels under basal conditions. B) Mitochondrial membrane potential of GFP- and Tau/APP-expressing cells untreated (CT) or reversibly treated with CCCP $(20 \mu \mathrm{M})$ for $6 \mathrm{~h}$ and then allowed to recover for $1 \mathrm{~h}$ (reversible CCCP), or treated for $7 \mathrm{~h}$ with CCCP (total CCCP). $(n=3$ independent experiments; $\mathrm{n} . \mathrm{s}$ : not significant, $\left.{ }^{*} p<0.05\right)$.

suggests a possible cause of mitochondrial dynamics alteration and synaptic damage [41]. In addition, the microtubule-directed traffic is a main player in the regulation of autophagy. Altered microtubule assembly impairs microtubule-dependent mitochondrial and autophagosomal transport toward lysosomes [42]. It is known that $A \beta$ induces a reduction in motile mitochondria [43] and hyperphosphorylated tau may block the transport of mitochondria and may disrupt the microtubule network trafficking leading to energy deprivation and oxidative stress [44].

Presently, we have analyzed the mitochondrial recycling process after the overexpression of $A P P$ and Tau in a human line of fibroblasts. We have demonstrated that increase of $A \beta P P$ or tau proteins generates an autophagy degradation phase activation correlating with higher lysosomal activity. The combination of AßPP and tau markedly enhanced autophagy flux by increasing AV synthesis and degradation. It might be reasonable to speculate that the boosting of autophagy flux may be a consequence of the proteotoxic stress generated by the overexpression of proteins that are prone to aggregate $[45,46]$.

The increased mitochondrial content observed in $A P P$ - or Tau- and APP/Tau-expressing cells suggested a compromised mitophagy in all cases, although there are some differences. In the case of $A P P$ - or Tau-expressing cells, we hypothesize that increased levels of PARK2 and PINK1 could be explained as an attempt to increase the mitochondrial recycling system. However, the diminished targeting of PARK2 and PINK1 to mitochondria may reflect either an improvement of the recycling of mitochondria by the increased autophagy degradation phase observed or an insufficient labelling of depolarized mitochondria to be degraded. In this regard, enhanced levels of $\Delta 1$-PINK1 may play an inhibitory effect over PARK2 mitochondrial translocation contributing to defective label of damage mitochondria [29]. Nevertheless, the study of mitochondrial $\Delta \Psi \mathrm{m}$ recovery after CCCP insult indicates that a main part of mitochondria is able to be recycled properly, although not as efficiently as control cells, which may explain the slight the long-term accumulation of mitochondria and FL-PINK. On the other hand, the combination of APP and Tau overexpression exhibited a dramatic accumulation of PINK1 in mitochondria after an insult but the recruitment of PARK2 to the mitochondria was decreased resulting in a defective labeling of damaged mitochondria for their removal, demonstrating a deficient crosstalk between these two proteins. This resulted in an impaired $\Delta \Psi \mathrm{m}$ recovery after CCCP challenge correlating with a major accumulation of dysfunctional mitochondria labeled by PINK1. This data together demonstrate a worsening of mitophagy failure due to the 
presence of tau. According to our results, it has been recently described that tau accumulation may induce mitophagy deficits by reducing level of PARK2 in the mitochondrial fraction [47, 48]. Moreover, diffuse forms of tau have been demonstrated to trap PARK2 in the cytosol, preventing its translocation to mitochondria [48]. This finding may explain part of mitophagy impairment that we observed. However, we have also shown inhibition of PARK2 translocation to mitochondria in $A P P$ overexpressing cells as well as a mitophagy failure aggravation in APP/Tau combination with respect to Tau. Therefore, further studies will be necessary to unravel the inhibitory role of A $\beta P P$ in mitophagy process and PARK2 translocation to mitochondria. Finally, to explain tau contribution to mitophagy failure, there are two factors that should be taken into consideration: 1) The deterioration of mitochondrial function caused by the deregulation of mitochondrial respiratory chain complexes $\mathrm{V}$ and I $[49,50]$ as well as by the blockage of mitochondrial pores through VDAC1 interaction [51] 2) The mitochondrial dynamics alteration by tau interaction with the fission protein DLP1 [41] accompanied with the interference of mitochondrial axonal transport by competition with their binding site to microtubules [52]. In conclusion, further studies will be necessary to fully explain the role of A $\beta P P$ and tau in the puzzle of mitophagy failure in $\mathrm{AD}$.

We have been able to demonstrate mitophagy impairment by overexpression of the two proteins mainly involved in AD. While this is a proof of concept and may help to understand the mechanisms involved in this pathology, this is only the simplest model with putative artifacts generated by supraphysiological expression levels that need to be ruled out. However, our previous findings of similar mitophagy failure in unmodified fibroblasts and brain samples from sporadic AD patients [14] corroborates the relevance of the conclusions taking in consideration that A $\beta P P$ and tau pathology is a common feature in sporadic and familiar cases of the disease.

In summary, we have demonstrated that the presence of tau aggravates the defect exhibited by APP overexpression leading to a clear mitophagy failure shown by unachievable recovery of mitochondrial membrane potential after an insult that results in the accumulation of dysfunctional mitochondria. This data supports the previously established idea that tau contribution is fundamental for AD-related pathology [53].

\section{ACKNOWLEDGMENTS}

We would like to acknowledge Dr. Jason Jon Fritz (Emory University School of Medicine, Atlanta GA) for the lentivector encoding APP and Prof. Kenneth S. Kosik (UC Santa Barbara, CA) for Tau lentivector. PHF1 and Tau 7.51 antibodies were kindly ceded by Dr. Peter Davies (Albert Einstein Coll., Bronx, NY, USA) and Dr. Claude M. Wischik (MRC, Cambridge, UK), respectively.

This work was supported by the Ministerio de Economía y Competitividad (SAF 2011 program, project 24841 and grant BES-2012-055068) from Spain and the Centro de Investigación en Red de Enfermedades Neurodegenerativas (CIBERNED, ISCIII, JA). R.G. has been funded by the AECC Scientific Foundation. The funders had no role in study design, data collection and analysis, decision to publish, or preparation of the manuscript. The authors would like to specially acknowledge Michael Lifvergren for English editing.

Authors' disclosures available online (https:// www.j-alz.com/manuscript-disclosures/19-0086r1).

\section{SUPPLEMENTARY MATERIAL}

The supplementary material is available in the electronic version of this article: http://dx.doi.org/ 10.3233/JAD-190086.

\section{REFERENCES}

[1] Masters CL, Multhaup G, Simms G, Pottgiesser J, Martins RN, Beyreuther K (1985) Neuronal origin of a cerebral amyloid: Neurofibrillary tangles of Alzheimer's disease contain the same protein as the amyloid of plaque cores and blood vessels. EMBO J 4, 2757-2763.

[2] Selkoe DJ (2001) Alzheimer's disease: Genes, proteins, and therapy. Physiol Rev 81, 741-766.

[3] Citron M, Diehl TS, Gordon G, Biere AL, Seubert P, Selkoe DJ (1996) Evidence that the 42- and 40-amino acid forms of amyloid beta protein are generated from the beta-amyloid precursor protein by different protease activities. Proc Natl Acad Sci U S A 93, 13170-13175.

[4] Neve RL, McPhie DL, Chen Y (2000) Alzheimer's disease: A dysfunction of the amyloid precursor protein(1). Brain Res 886, 54-66.

[5] Avila J, Leon-Espinosa G, Garcia E, Garcia-Escudero V, Hernandez F, Defelipe J (2012) Tau phosphorylation by GSK3 in different conditions. Int $J$ Alzheimers Dis 2012, 578373.

[6] Hanger DP, Anderton BH, Noble W (2009) Tau phosphorylation: The therapeutic challenge for neurodegenerative disease. Trends Mol Med 15, 112-119.

[7] Selkoe DJ (2000) Toward a comprehensive theory for Alzheimer's disease. Hypothesis: Alzheimer's disease is caused by the cerebral accumulation and cytotoxicity of 
amyloid beta-protein. Ann N Y Acad Sci 924, 17-25.

[8] Nixon RA, Wegiel J, Kumar A, Yu WH, Peterhoff C, Cataldo A, Cuervo AM (2005) Extensive involvement of autophagy in Alzheimer disease: An immuno-electron microscopy study. J Neuropathol Exp Neurol 64, 113-122.

[9] Kuma A, Hatano M, Matsui M, Yamamoto A, Nakaya H, Yoshimori T, Ohsumi Y, Tokuhisa T, Mizushima N (2004) The role of autophagy during the early neonatal starvation period. Nature 432, 1032-1036.

[10] Klionsky DJ, Emr SD (2000) Autophagy as a regulated pathway of cellular degradation. Science 290, 1717-1721.

[11] Jahreiss L, Menzies FM, Rubinsztein DC (2008) The itinerary of autophagosomes: From peripheral formation to kiss-and-run fusion with lysosomes. Traffic 9, 574-587.

[12] Scheper W, Nijholt DA, Hoozemans JJ (2011) The unfolded protein response and proteostasis in Alzheimer disease: Preferential activation of autophagy by endoplasmic reticulum stress. Autophagy 7, 910-911.

[13] Garcia-Escudero V, Martin-Maestro P, Perry G, Avila J (2013) Deconstructing mitochondrial dysfunction in Alzheimer disease. Oxid Med Cell Longev 2013, 162152.

[14] Martin-Maestro P, Gargini R, Perry G, Avila J, GarciaEscudero V (2016) PARK2 enhancement is able to compensate mitophagy alterations found in sporadic Alzheimer's disease. Hum Mol Genet 25, 792-806.

[15] Moreira PI, Siedlak SL, Wang X, Santos MS, Oliveira CR, Tabaton M, Nunomura A, Szweda LI, Aliev G, Smith MA, Zhu X, Perry G (2007) Autophagocytosis of mitochondria is prominent in Alzheimer disease. J Neuropathol Exp Neurol 66, 525-532.

[16] Moreira PI, Siedlak SL, Wang X, Santos MS, Oliveira CR, Tabaton M, Nunomura A, Szweda LI, Aliev G, Smith MA, Zhu X, Perry G (2007) Increased autophagic degradation of mitochondria in Alzheimer disease. Autophagy 3, 614-615.

[17] Otvos Jr L, Feiner L, Lang E, Szendrei G, Goedert M, Lee VMY (1994) Monoclonal antibody PHF-1 recognizes tau protein phosphorylated at serine residues 396 and 404. J Neurosci Res 39, 669-673.

[18] Novak M, Jakes R, Edwards PC, Milstein C, Wischik CM (1991) Difference between the tau protein of Alzheimer paired helical filament core and normal tau revealed by epitope analysis of monoclonal antibodies 423 and 7.51. Proc Natl Acad Sci U S A 88, 5837-5841.

[19] Klionsky DJ, Abdelmohsen K, Abe A, Abedin MJ, Abeliovich $\mathrm{H}$, Acevedo Arozena A, Adachi H, Adams CM, Adams PD, Adeli K, et al. (2016) Guidelines for the use and interpretation of assays for monitoring autophagy (3rd edition). Autophagy 12, 1-222.

[20] Rubinsztein DC, Cuervo AM, Ravikumar B, Sarkar S, Korolchuk V, Kaushik S, Klionsky DJ (2009) In search of an "autophagomometer". Autophagy 5, 585-589.

[21] Agca C, Fritz JJ, Walker LC, Levey AI, Chan AW, Lah JJ, Agca Y (2008) Development of transgenic rats producing human beta-amyloid precursor protein as a model for Alzheimer's disease: Transgene and endogenous APP genes are regulated tissue-specifically. BMC Neurosci 9, 28.

[22] Uetsuki T, Takemoto K, Nishimura I, Okamoto M, Niinobe M, Momoi T, Miura M, Yoshikawa K (1999) Activation of neuronal caspase- 3 by intracellular accumulation of wildtype Alzheimer amyloid precursor protein. J Neurosci 19, 6955-6964.

[23] Cataldo AM, Nixon RA (1990) Enzymatically active lysosomal proteases are associated with amyloid deposits in Alzheimer brain. Proc Natl Acad Sci U S A 87, 3861-3865.
[24] Cataldo AM, Barnett JL, Pieroni C, Nixon RA (1997) Increased neuronal endocytosis and protease delivery to early endosomes in sporadic Alzheimer's disease: Neuropathologic evidence for a mechanism of increased beta-amyloidogenesis. J Neurosci 17, 6142-6151.

[25] Ding WX, Yin XM (2012) Mitophagy: Mechanisms, pathophysiological roles, and analysis. Biol Chem 393, 547-564.

[26] Narendra D, Tanaka A, Suen DF, Youle RJ (2008) Parkin is recruited selectively to impaired mitochondria and promotes their autophagy. J Cell Biol 183, 795-803.

[27] Vives-Bauza C, Zhou C, Huang Y, Cui M, de Vries RL, Kim J, May J, Tocilescu MA, Liu W, Ko HS, Magrane J, Moore DJ, Dawson VL, Grailhe R, Dawson TM, Li C, Tieu K, Przedborski S (2010) PINK1-dependent recruitment of Parkin to mitochondria in mitophagy. Proc Natl Acad Sci U S A 107, 378-383.

[28] Narendra DP, Jin SM, Tanaka A, Suen DF, Gautier CA, Shen J, Cookson MR, Youle RJ (2010) PINK1 is selectively stabilized on impaired mitochondria to activate Parkin. PLoS Biol 8, e1000298.

[29] Fedorowicz MA, de Vries-Schneider RL, Rub C, Becker D, Huang Y, Zhou C, Alessi Wolken DM, Voos W, Liu Y, Przedborski S (2014) Cytosolic cleaved PINK1 represses Parkin translocation to mitochondria and mitophagy. EMBO Rep 15, 86-93.

[30] Martinez-Vicente M, Talloczy Z, Wong E, Tang G, Koga H, Kaushik S, de Vries R, Arias E, Harris S, Sulzer D, Cuervo $\mathrm{AM}$ (2010) Cargo recognition failure is responsible for inefficient autophagy in Huntington's disease. Nat Neurosci 13, 567-576.

[31] Katsuragi Y, Ichimura Y, Komatsu M (2015) p62/SQSTM1 functions as a signaling hub and an autophagy adaptor. FEBS J 282, 4672-4678.

[32] Witte ME, Bol JG, Gerritsen WH, van der Valk P, Drukarch B, van Horssen J, Wilhelmus MM (2009) Parkinson's disease-associated parkin colocalizes with Alzheimer's disease and multiple sclerosis brain lesions. Neurobiol Dis 36, 445-452.

[33] Geisler S, Holmström KM, Skujat D, Fiesel FC, Rothfuss OC, Kahle PJ, Springer W (2010) PINK1/Parkin-mediated mitophagy is dependent on VDAC1 and p62/SQSTM1. Nat Cell Biol 12, 119

[34] Matsuda N, Sato S, Shiba K, Okatsu K, Saisho K, Gautier CA, Sou Y-s, Saiki S, Kawajiri S, Sato F, Kimura M, Komatsu M, Hattori N, Tanaka K (2010) PINK1 stabilized by mitochondrial depolarization recruits Parkin to damaged mitochondria and activates latent Parkin for mitophagy. J Cell Biol 189, 211-221.

[35] Martín-Maestro P, Gargini R, Perry G, Avila J, GarcíaEscudero V (2015) PARK2 enhancement is able to compensate mitophagy alterations found in sporadic Alzheimer's disease. Hum Mol Genet 25, 792-806.

[36] Nunomura A, Perry G, Aliev G, Hirai K, Takeda A, Balraj EK, Jones PK, Ghanbari H, Wataya T, Shimohama S, Chiba S, Atwood CS, Petersen RB, Smith MA (2001) Oxidative damage is the earliest event in Alzheimer disease. $\mathrm{J} \mathrm{Neu-}$ ropathol Exp Neurol 60, 759-767.

[37] Moreira PI, Cardoso SM, Santos MS, Oliveira CR (2006) The key role of mitochondria in Alzheimer's disease. J Alzheimers Dis 9, 101-110.

[38] Casadesus G, Smith MA, Basu S, Hua J, Capobianco DE, Siedlak SL, Zhu X, Perry G (2007) Increased isoprostane and prostaglandin are prominent in neurons in Alzheimer disease. Mol Neurodegener 2, 2. 
[39] Moreira PI, Harris PL, Zhu X, Santos MS, Oliveira CR, Smith MA, Perry G (2007) Lipoic acid and N-acetyl cysteine decrease mitochondrial-related oxidative stress in Alzheimer disease patient fibroblasts. J Alzheimers Dis 12, 195-206.

[40] Xu Z, Klionsky DJ (2016) Autophagy promotes cell motility by driving focal adhesion turnover. Autophagy 12, 16851686.

[41] Manczak M, Calkins MJ, Reddy PH (2011) Impaired mitochondrial dynamics and abnormal interaction of amyloid beta with mitochondrial protein Drp1 in neurons from patients with Alzheimer's disease: Implications for neuronal damage. Hum Mol Genet 20, 2495-2509.

[42] Arduino DM, Esteves AR, Cardoso SM (2013) Mitochondria drive autophagy pathology via microtubule disassembly: A new hypothesis for Parkinson disease. Autophagy 9, 112-114.

[43] Rui Y, Tiwari P, Xie Z, Zheng JQ (2006) Acute impairment of mitochondrial trafficking by beta-amyloid peptides in hippocampal neurons. J Neurosci 26, 10480-10487.

[44] Stamer K, Vogel R, Thies E, Mandelkow E, Mandelkow EM (2002) Tau blocks traffic of organelles, neurofilaments, and APP vesicles in neurons and enhances oxidative stress. $J$ Cell Biol 156, 1051-1063.

[45] Bandyopadhyay B, Li G, Yin H, Kuret J (2007) Tau aggregation and toxicity in a cell culture model of tauopathy. $J$ Biol Chem 282, 16454-16464.

[46] Spires TL, Meyer-Luehmann M, Stern EA, McLean PJ, Skoch J, Nguyen PT, Bacskai BJ, Hyman BT (2005) Dendritic spine abnormalities in amyloid precursor protein transgenic mice demonstrated by gene transfer and intravital multiphoton microscopy. J Neurosci 25, 7278-7287.

[47] Hu Y, Li XC, Wang ZH, Luo Y, Zhang X, Liu XP, Feng Q, Wang Q, Yue Z, Chen Z, Ye K, Wang JZ, Liu GP (2016)
Tau accumulation impairs mitophagy via increasing mitochondrial membrane potential and reducing mitochondrial parkin. Oncotarget 7, 17356-17368.

[48] Cummins N, Tweedie A, Zuryn S, Bertran-Gonzalez J, Götz J (2018) Disease-associated tau impairs mitophagy by inhibiting Parkin translocation to mitochondria. EMBO J 38, e99360.

[49] David DC, Hauptmann S, Scherping I, Schuessel K, Keil U, Rizzu P, Ravid R, Drose S, Brandt U, Muller WE, Eckert A, Gotz J (2005) Proteomic and functional analyses reveal a mitochondrial dysfunction in P301L tau transgenic mice. J Biol Chem 280, 23802-23814.

[50] Schulz KL, Eckert A, Rhein V, Mai S, Haase W, Reichert AS, Jendrach M, Muller WE, Leuner K (2012) A new link to mitochondrial impairment in tauopathies. Mol Neurobiol 46, 205-216.

[51] Manczak M, Reddy PH (2012) Abnormal interaction of VDAC1 with amyloid beta and phosphorylated tau causes mitochondrial dysfunction in Alzheimer's disease. Hum Mol Genet 21, 5131-5146.

[52] Hagiwara H, Yorifuji H, Sato-Yoshitake R, Hirokawa N (1994) Competition between motor molecules (kinesin and cytoplasmic dynein) and fibrous microtubule-associated proteins in binding to microtubules. J Biol Chem 269, 35813589.

[53] Saera-Vila A, Kish PE, Louie KW, Grzegorski SJ, Klionsky DJ, Kahana A (2016) Autophagy regulates cytoplasmic remodeling during cell reprogramming in a zebrafish model of muscle regeneration. Autophagy 12, 1864-1875. 